\title{
Syntheses of chondroitin sulfate tetrasaccharide structures containing 4,6-disulfate patterns and analysis of their interaction with glycosaminoglycan-binding protein
}

\author{
Kento Miyachi, ${ }^{1}$ Masahiro Wakao, ${ }^{1 *}$ Yasuo Suda ${ }^{1,2} *$ \\ ${ }^{1}$ Department of Chemistry, Biotechnology, and Chemical Engineering, Graduate School of \\ Science and Engineering, Kagoshima University, 1-21-40 Kohrimoto, Kagoshima 890-0065, \\ Japan \\ ${ }^{2}$ SUDx-Biotec Corporation, 1-41-1 Shiroyama, Kagoshima 890-0013, Japan
}

\section{AUTHOR EMAIL ADDRESS}

wakao@eng.kagoshima-u.ac.jp, ysuda@eng.kagoshima-u.ac.jp

AUTHOR PHONE\&FAX

TEL: (+81)99-285-7843, FAX: (+81)99-285-7856 for M. Wakao

TEL \& FAX: (+81)99-285-8369 for Y. Suda 


\begin{abstract}
Chondroitin sulfate tetrasaccharide ligand conjugates, namely

GlcA-GalNAc6S-GlcA-GalNAc4S6S (CS-C+E) 1,

GlcA2S-GalNAc6S-GlcA2S-GalNAc4S6S (CS-D+T) 2,

GlcA-GalNAc4S6S-GlcA-GalNAc4S (CS-E+A) 3, GlcA-GalNAc4S6S-GlcA-GalNAc6S

(CS-E+C) 4, and GlcA-GalNAc4S6S-GlcA-GalNAc4S6S (CS-E+E) 5, were systematically

synthesized using a disaccharide building block 6. Synthesized CS tetrasaccharide structures were immobilized onto gold-coated chips to prepare array-type sugar chips, and the binding properties of protein were evaluated by surface plasmon resonance imaging biosensor.

CS-D+T, CS-E+A, CS-E+C, and CS-E+E showed greater affinity for basic fibroblast growth factor than did other tetrasaccharides $(C S-C+D, C+E, D+D)$.
\end{abstract}

\title{
Keyword
}

basic fibroblast growth factor; chondroitin sulfate; glycosaminoglycan; sugar chips; surface plasmon resonance; synthesis 


\section{Text}

Chondroitin sulfate (CS) is a glycosaminoglycan (GAG) that is widely distributed throughout various tissues as components of the cell membrane or extracellular matrix. CS possesses a repeating disaccharide structure that is composed of glucuronic acid (GlcA) and $N$-acetylgalactosamine (GalNAc), and it is often sulfated heterogeneously by multiple and random enzymatic modifications during its biosynthesis. ${ }^{1}$ The structure of CS is classified on the basis of the disaccharide unit; type A (CS-A), type C (CS-C), type D (CS-D), and type E (CS-E) units are the major disaccharide structures known from natural sources to date. CS-E, containing a 4, 6-disulfated GalNAc moiety, interacts with various bioactive proteins, such as growth factors, cytokines, and extracellular matrix proteins, and regulates their biofuctions. ${ }^{2-8}$ Therefore, the structure-activity relationship of the CS-E unit at the molecular level has been intensively studied to gain understanding of its biofunction.

Although much effort ${ }^{9-22}$ has been invested to obtain a structurally defined CS-E oligosaccharide, the synthesis of oligosaccharides containing a CS-E unit is still challenging. In particular, the synthesis of a hetero-dimeric CS tetrasaccharide structure has not been reported. In this study, we attempted the systematic synthesis of CS tetrasaccharide ligand conjugates containing homo- or hetero-dimeric CS-E structures and their analogs. Additionally, we used surface plasmon resonance (SPR) imaging to investigate the binding properties of CS-binding proteins using the tetrasaccharide structures we had synthesized.

For efficient syntheses of the CS tetrasaccharide structures 1-5 (Figure 1), trifluoroimidates $\mathbf{9}$ and $\mathbf{1 3}$ were prepared as glycosyl donors; these compounds were utilized for the generation of CS-C and -D and CS-E units, respectively (Scheme 1). Trifluoroimidate 9 was induced by selective ring-opening of the benzylidene group, whereas trifluoroimidate 13 was induced by acidic hydrolysis of the benzylidene group. For both compounds, the 
induction was followed by levunylation from the disaccharide building block $\mathbf{6}$.

Synthesis of the acceptor moiety was carried out as shown in Scheme 2. Three kinds of acceptor moieties were derived from a common trisaccharide intermediate 14. Desilylation of the 4" position yielded acceptor 15, which was used for the construction of the CS-E and -T units. After selective ring-opening of the benzylidene group and levulinoylation, removal of the tert-butyldimethylsilyl (TBDMS) group yielded acceptors $\mathbf{1 8}$ and 20, which were used for construction of the CS-A and CS-C units, respectively.

With these glycosyl donors and acceptors, we then attempted the synthesis of pentasaccharides and their ligand conjugates (Scheme 3). Glycosylation with an appropriate combination of glycosyl donor and acceptor was carried out in the presence of trimethylsilyl trifluoromethanesulfonate (TMSOTf) to produce the corresponding pentasaccharides. The pentasaccharides so obtained were then converted into CS tetrasaccharide structures and their ligand conjugates via a suitable deprotection and sulfation protocol. Pentasaccharides 21, 22, 23, and 24 were treated with a $\mathrm{Zn}-\mathrm{Cu}$ couple in $\mathrm{AcOH}$, and the amino groups were acetylated. Selective deprotection of the levulinoyl (Lev) group was performed by using hydrazine hydrate in the presence of AcOH. The benzoyl (Bz) groups and methyl esters were hydrolyzed under basic conditions (with $\mathrm{NaOH}$ ). The resulting free hydroxyl groups were sulfated with a sulfur trioxide-pyridine complex at $50{ }^{\circ} \mathrm{C}$. After deprotection of the TBDMS group with HF•pyridine, all of the benzyl protective groups were removed by hydrogenolysis with a catalytic amount of Pd/C. Pentasaccharides 25, 26, 27, 28, and 29 were purified by ODS column chromatography, followed by gel filtration chromatography with Sephadex G-10 or G-15 fine to produce the desired pentasaccharides containing any of the following two disaccharide units: CS-C+E, CS-D+T, CS-E+A, CS-E+C, or CS-E+E. Ligand conjugates were synthesized with these pentasaccharides, according to previously described methods, ${ }^{23,24}$ 
yielding CS-tetrasaccharide ligand conjugates 1, 2, 3, 4, and 5. These compounds were purified by ODS chromatography and gel filtration chromatography, using Sephadex G-10 or G-15 fine, and their structures were confirmed by ${ }^{1} \mathrm{H}$ NMR and ESI-TOF/MS analyses.

Binding interactions were investigated using an SPR imaging biosensor, which allows real-time analysis of interactions without requiring labeling of analytes. Basic fibroblast growth factor (FGF-2) was chosen as the GAG-protein, because FGF-2 is known to interact strongly with the CS-E structure; we therefore investigated the structure-activity relationship of the CS-E unit-containing microdomain structure in detail. Specific binding interactions were clearly observed with ligand-conjugates containing the CS-T disaccharide ${ }^{25}$, $\mathrm{CS}-\mathrm{E}+\mathrm{A}, \mathrm{CS}-\mathrm{E}+\mathrm{C}, \mathrm{CS}-\mathrm{E}+\mathrm{D}^{26}$, and $\mathrm{CS}-\mathrm{E}+\mathrm{E}$ tetrasaccharides (Figure 2a). The kinetic parameters of these interactions are summarized in Table 1. It has been reported that FGF-2 is strongly recognized by tetrasaccharides containing CS-E unit structures. ${ }^{27-32}$ To characterize the properties of CS tetrasaccharide structures in greater detail, sugar chips, immobilized with a mixture of CS ligand-conjugates prepared in this study and with a Glc ligand-conjugate $3^{23,33}$ which does not bind FGF-2 (Figure 2b: 1:2 molar ratio, Figure 2c: 1:8 molar ratio), were fabricated and tested. FGF-2 bound strongly to the CS-T disaccharide and CS-D+T, $-E+A,-E+C,-E+D$, and $-E+E$ tetrasaccharides (Figure 2b, 2c). The binding affinity of the CS tetrasaccharide structures differed markedly, depending on whether they had a CS-E unit $(C S-E+A,-E+C,-E+D$, and $-E+E)$ or a CS-D unit $\left(C S-D+D^{26}, C S-D+T\right)$ at the non-reducing end, This indicated that the CS disaccharide unit at the reducing end was also recognized by the protein. Furthermore, our sugar chip technique showed that the inverse sequence had different binding properties; the CS-E+C tetrasaccharide interacted strongly with FGF-2, whereas the CS-C+E tetrasaccharide did not.

In conclusion, we synthesized five types of homo- and hetero-dimeric CS 
tetrasaccharide structures with a 4,6-disulfated pattern, viz., CS-C+E 1, CS-D+T 2, CS-E+A 3, CS-E+C 4, and CS-E+E 5. In addition, we fabricated sugar chips with these synthesized CS ligand-conjugates and analyzed their binding interaction with FGF-2. The results showed that our sugar chip technology can be utilized for real-time analysis of the structure-function relationship of GAG-binding proteins at the molecular level in real-time, without requiring labeling of analytes.. Although construction of a CS tetrasaccharide library is underway, our CS sugar chips may be used for high-throughput analysis of the binding properties of protein samples in order to decipher the CS sugar code. 


\section{Acknowledgements}

The present study was financially supported in part by grants from the Ministry of

Education, Culture, Sports, Science, and Technology (MEXT; Grant-in-Aid for Young

Scientists B, Kakenhi 21710233, to MW and Grant-in-Aid for Scientific Research B, Kakenhi

23300356, to YS), Japan Science and Technology Agency (JST) (A-Step, Post-venture

Support, AS2416907P to YS), and Japan Ministry for Health, Labor, and Welfare

(Nano-Medicine, H21-nano-ippan-002 to YS). 


\section{References}

1. Silbert, J. E.; Sugumaran, G. IUBMB Life 2002, 54, 177.

2. Ueoka, C.; Kaneda, N.; Okazaki, I.; Nadanaka, S.; Muramatsu, T.; Sugahara, K. J. Biol. Chem. 2000, 277, 37407.

3. Takagaki, K.; Munakata, H.; Kakizaki, I.; Iwafune, M.; Itabashi, T.; Endo, M. J. Biol. Chem. 2002, 277, 8882.

4. Kawashima, H.; Atarashi, K.; Hirose, M.; Hirose, J.; Yamada, S.; Sugahara, K.; Miyasaka, M. J. Biol. Chem. 2002, 277, 12921.

5. Deepa, S.S.; Umehara, Y.; Higashiyama, S.; Itoh, N.; Sugahara, K. J. Biol. Chem. 2002, $277,43707$.

6. Sugahara, K.; Mikami, T.; Uyama, T.; Mizuguchi, S.; Nomura, K.; Kitagawa, H. Curr. Opin. Chem. Biol. 2003, 13, 612.

7. Hashiguchi, T.; Mizumoto, S.; Yamada, S.; Sugahara, K. Glycoconj. J. 2010, 27, 49.

8. Mizumoto, S.; Fongmoon, D.; Sugahara, K. Glycoconj. J. 2013, 30, 619.

9. Jacquinet, J. C. Carbohydr. Res. 1990, 199, 153.

10. Lubineau, A.; Bonnaffé, D. Eur. J. Org. Chem. 1999, 2523.

11. Fujikawa, S.; Ohmae, M.; Kobayashi S. Biomacromol. 2005, 6, 2935.

12. Jacquinet, J.C.; Lopin-Bon, C.; Vibert, A. Chem. Eur. J. 2009, 15, 9579.

13. Tamura, J.; Neumann, K.W.; Ogawa, T. Bioorg. Med. Chem. Lett. 1995, 5, 1351.

14. Tamura, J.; Neumann, K. W.; Kurono, S.; Ogawa, T. Carbohydr. Res. 1998, 305, 43.

15. Karst, N.; Jacquinet, J.C. Eur. J. Org. Chem. 2002, 815.

16. Tully, S. E.; Mabon, R.; Gama, C.I.; Tsai, S.M.; Liu, X.; Hsieh-Wilson, L. J. Am. Chem. 
Soc. 2004, 126, 7736.

17. Gama, C.I.; Tully, S.E.; Sotogaku, N.; Clark, P.M.; Rawat, M.; Vaidehi, N.; Goddard, W. A., III.; Nishi, A.; Hsieh-Wilson, L.C. Nat. Chem. Biol. 2006, 2, 467.

18. Tamura, J.; Tsutsumisita-Nakai, N.; Nakao, Y.; Kawano, M.; Kato, S.; Takeda, N.; Nadanaka, S.; Kitagawa, H. Bioorg. Med. Chem. Lett. 2012, 22, 1371.

19. Bedini, E.; Castro, C.D.; De Rosa, M.; Di Nola, A.; Restaino, O.F.; Schiraldi, C.; Parrilli, M. Chem. Eur. J. 2012, 18, 2123.

20. Maza, S.; Mar Kayser, M.; Macchione, G.; López-Prados, J.; Angulo, J.; de Paz, J.L.; Nieto, P.M. Org. Biomol. Chem. 2013, 42, 3510.

21. Macchione, G.; Maza, S.; Mar Kayser, M.; de Paz, J. L.; Nieto, P. M. Eur. J. Org. Chem. 2014, 3868.

22. Jacquinet, J. C.; Lopin-Bon, C. Carbohydr. Res. 2015, 402, 35.

23. Suda, Y.; Arano, A.; Fukui, Y.; Koshida, S.; Wakao, M.; Nishimura, T.; Kusumoto, S.; Sobel, M. Bioconjugate Chem. 2006, 17, 1125.

24. Wakao, M.; Saito, A.; Ohishi, K.; Kishimoto, Y.; Nishimura, T.; Sobel, M.; Suda, Y. Bioorg. Med. Chem. Lett. 2008, 18, 2499.

25. Wakao, M.; Obata, R.; Miyachi, K.; Kakitsubata, Y.; Kondo, T.; Sakami, C.; Suda, Y. submitted.

26. Miyachi, K.; Wakao, M.; Ichiki, Y.; Sakami, C.; Suda, Y. submitted.

27. Turnbell, J.E.; Fernig, D.G.; Ke, Y.; Wilkinson, M.C.; Gallagher, J.T. J. Biol. Chem. 1992, 267, 10337.

28. Habuchi, H.; Suzuki, S.; Saito, T.; Tamura, T.; Harada, T.; Yoshida, K.; Kimata, K. Biochem. J. 1992, 285, 805.

29. Nandini, C.D.; Mikami, T.; Ohta, M.; Itoh, N.; Akiyama-Nambu, F.; Sugahara, K. J. Biol. 
Chem. 2004, 279, 50799.

30. Bao, X.F.; Nishimura, S.; Mikami, T.; Yamada, S.; Itoh, N.; Sugahara, K. J. Biol. Chem. 2004, 279, 9765.

31. Shipp, E.L.; Hsieh-Wilson, L.C. Chem. Biol. 2007, 14, 195.

32. Rogers, C.J.; Clark, P. M.; Tully, S.E.; Abrol, R.; Garcia, K.C; Goddard III, W.A.; Hsieh-Wilson, L.C. Proc. Nat. Acad. Sci. 2011, 108, 9747.

33. Nakamura-Tsuruta, S.; Kishimoto, Y.; Nishimura, T.; Suda, Y. J. Biochem. 2008, 143, 833. 


\section{Legends to Schemes and Figures}

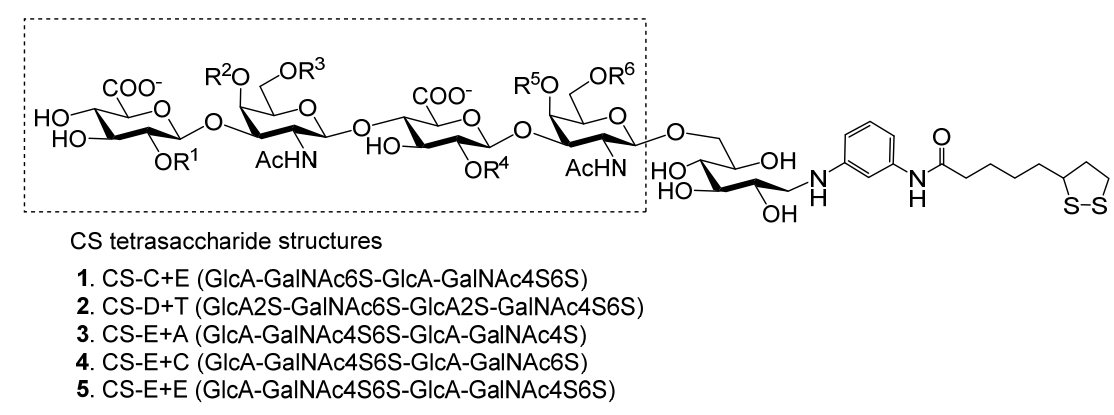

Figure 1. Tetrasaccharide structures 1-5. 


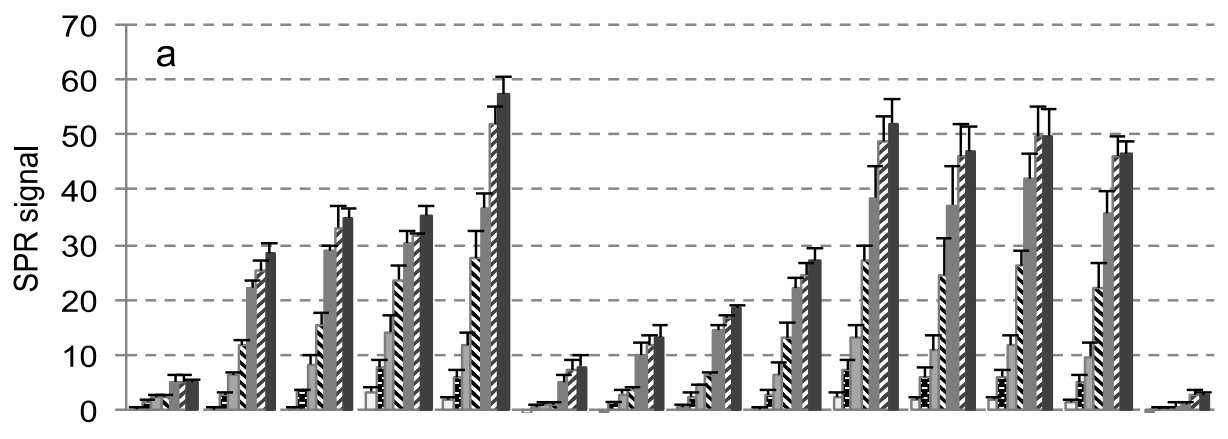

$$
\begin{aligned}
& \square 8 \mathrm{nM} \\
& \mathbf{8} 16 \mathrm{nM} \\
& \square 31 \mathrm{nM} \\
& \mathbf{\$} 63 \mathrm{nM} \\
& \square 125 \mathrm{nM} \\
& \square 250 \mathrm{nM} \\
& \square 500 \mathrm{nM}
\end{aligned}
$$
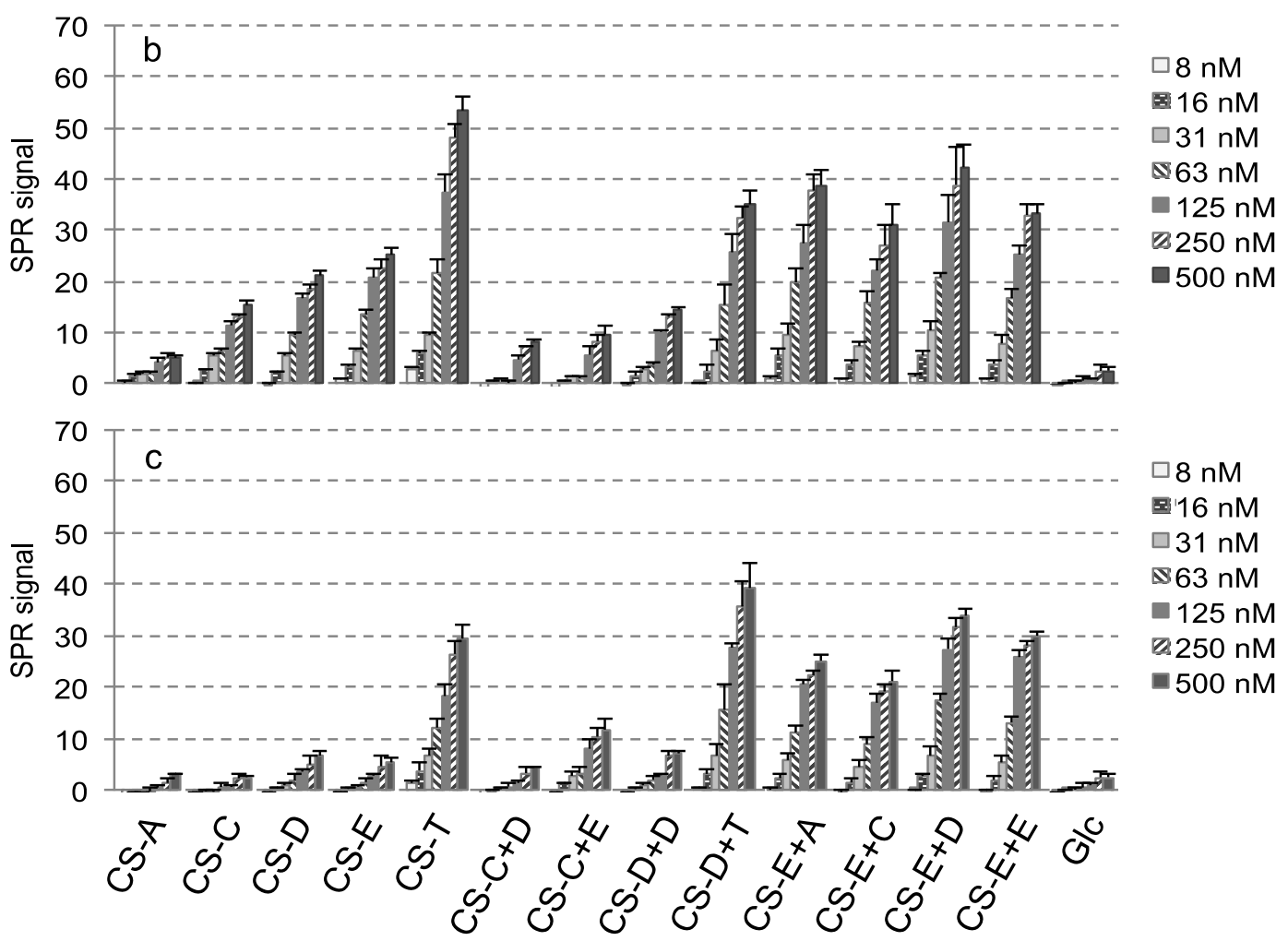

Figure 2. Binding study of FGF-2 and chondroitin sulfate (CS) subunits using SPR imaging. Measurements were carried out with analytes in the concentration range between 8 and 500 nM. Bar graph profiles of different concentrations of FGF-2 at the maxmum binding intensity of SPR imaging. The CS sugar chain and Glc ratio were (a) 1:0, (b) 1:2, and (c) 1:8. Abbreviations: CS-A = GlcA-GalNAc4S-Glc ligand conjugate 31 was immobilized; $\mathrm{CS}-\mathrm{C}=$ GlcA-GalNAc6S-Glc ligand conjugate 32 was immobilized; CS-D = GlcA2S-GalNAc6S-Glc 
ligand conjugate 33 was immobilized; CS-E = GlcA-GalNAc4S6S-Glc ligand conjugate 34 was immobilized; CS-T = GlcA2S-GalNAc4S6S-Glc ligand conjugate 35 was immobilized; CS-C+D = GlcA-GalNAc6S-GlcA2S-GalNAc6S-Glc ligand conjugate 36 was immobilized; CS-C+E = GlcA-GalNAc6S-GlcA-GalNAc4S6S-Glc ligand conjugate 1 was immobilized; $\mathrm{CS}-\mathrm{D}+\mathrm{D}=$ GlcA2S-GalNAc6S-GlcA2S-GalNAc6S-Glc ligand conjugate 37 was immobilized; CS-D+T = GlcA2S-GalNAc6S-GlcA2S-GalNAc4S6S-Glc ligand conjugate 2 was immobilized; CS-E+A = GlcA-GalNAc4S6S-GlcA-GalNAc4S-Glc ligand conjugate 3 was immobilized; CS-E+C = GlcA-GalNAc4S6S-GlcA-GalNAc6S-Glc ligand conjugate 4 was immobilized; CS-E+D = GlcA-GalNAc4S6S-GlcA2S-GalNAc6S-Glc ligand conjugate 38 was immobilized; CS-E+E= GlcA-GalNAc4S6S-GlcA-GalNAc4S6S-Glc ligand conjugate 5 was immobilized; Glc = Glc-Glc (maltose) ligand conjugate 39 was immobilized. 


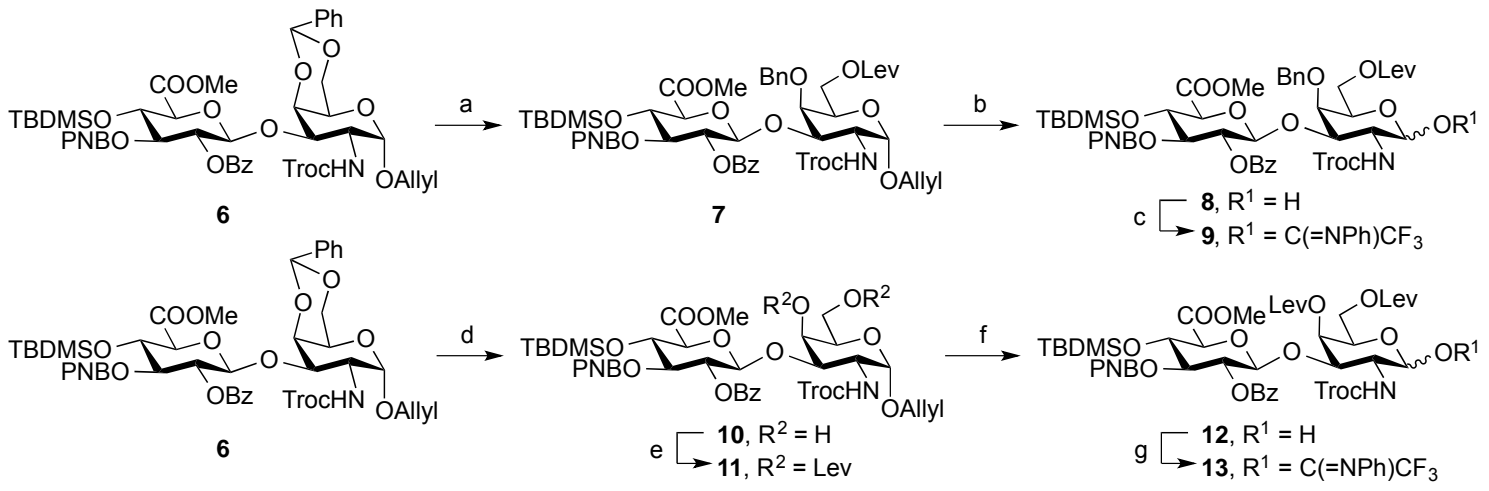

Scheme 1. Synthesis of glycosyl donor 9 and 13. (a) $\mathrm{Et}_{3} \mathrm{SiH}, \mathrm{PhBCl}_{2}$ in $\mathrm{CH}_{2} \mathrm{Cl}_{2},-78{ }^{\circ} \mathrm{C}, 10$ min; LevOH, DMAP, DCC in $\mathrm{CH}_{2} \mathrm{Cl}_{2}$, r.t., 2 h, 90\% (2 steps); (b) $\operatorname{Ir}\left[(\mathrm{cod})\left(\mathrm{PMePh}_{2}\right)_{2}\right] \mathrm{PF}_{6}, \mathrm{H}_{2}$ in THF, r.t., 30 min; $\mathrm{I}_{2}, \mathrm{H}_{2} \mathrm{O}$ in THF, r.t., 1 h, 93\% (2 steps); (c) $\mathrm{CF}_{3} \mathrm{C}(=\mathrm{NPh}) \mathrm{Cl}, \mathrm{K}_{2} \mathrm{CO}_{3}$ in $\mathrm{CH}_{2} \mathrm{Cl}_{2}, 18$ h; (d) TFA, $\mathrm{H}_{2} \mathrm{O}$ in $\mathrm{CH}_{2} \mathrm{Cl}_{2}, 0{ }^{\circ} \mathrm{C}, 2 \mathrm{~h}, 89 \%$; (e) LevOH, DMAP, DCC in $\mathrm{CH}_{2} \mathrm{Cl}_{2}$, r.t., 2 h, 97\%; (f) $\operatorname{Ir}\left[(\mathrm{cod})\left(\mathrm{PMePh}_{2}\right)_{2}\right] \mathrm{PF}_{6}, \mathrm{H}_{2}$ in THF, r.t., 30 min; $\mathrm{I}_{2}, \mathrm{H}_{2} \mathrm{O}$ in THF, r.t., 1 h, $98 \%$ (2 steps); (g) $\mathrm{CF}_{3} \mathrm{C}(=\mathrm{NPh}) \mathrm{Cl}, \mathrm{K}_{2} \mathrm{CO}_{3}$ in $\mathrm{CH}_{2} \mathrm{Cl}_{2}, 12$ h. 


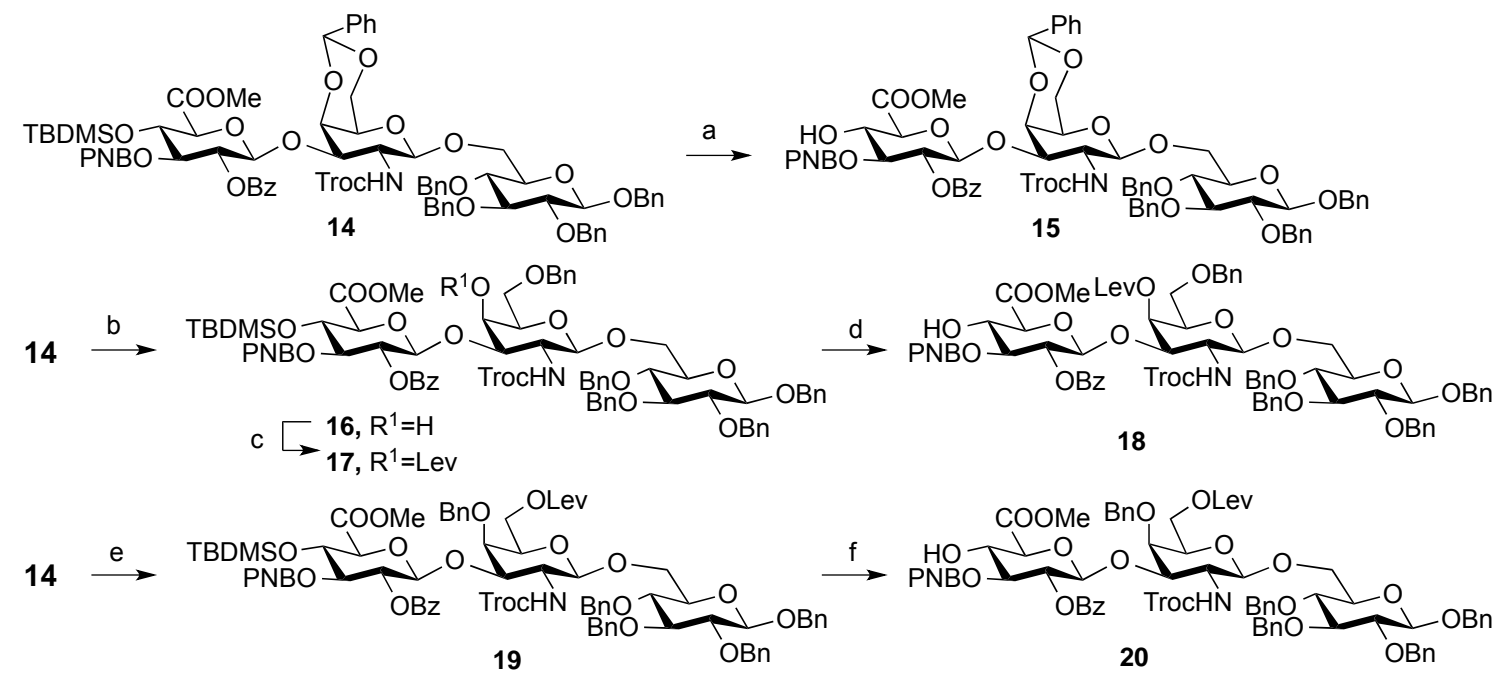

Scheme 2. Syntheses of glycosyl acceptor 15, 18, and 20.(a) HF•pyridine in THF, Pyr., $0{ }^{\circ} \mathrm{C}$ $\rightarrow$ r.t., 24 h, 97 \%; (b) $\mathrm{Et}_{3} \mathrm{SiH}$, TfOH in $\mathrm{CH}_{2} \mathrm{Cl}_{2},-78{ }^{\circ} \mathrm{C}, 10 \mathrm{~min}, 82 \%$; (c) LevOH, DMAP, DCC in $\mathrm{CH}_{2} \mathrm{Cl}_{2}$, r.t., 2 h, 84\%; (d) HF•pyridine in THF, Pyr., $0{ }^{\circ} \mathrm{C} \rightarrow$ r.t., 19 h, 94\%; (e) $\mathrm{Et}_{3} \mathrm{SiH}, \mathrm{PhBCl}_{2}$ in $\mathrm{CH}_{2} \mathrm{Cl}_{2},-78{ }^{\circ} \mathrm{C}, 10 \mathrm{~min}$; LevOH, DMAP, DCC in $\mathrm{CH}_{2} \mathrm{Cl}_{2}$, r.t., 2 h, $90 \%$ (2 steps); (f) HF•pyridine in THF, Pyr., $0{ }^{\circ} \mathrm{C} \rightarrow$ r.t., 24 h, 97\%. 


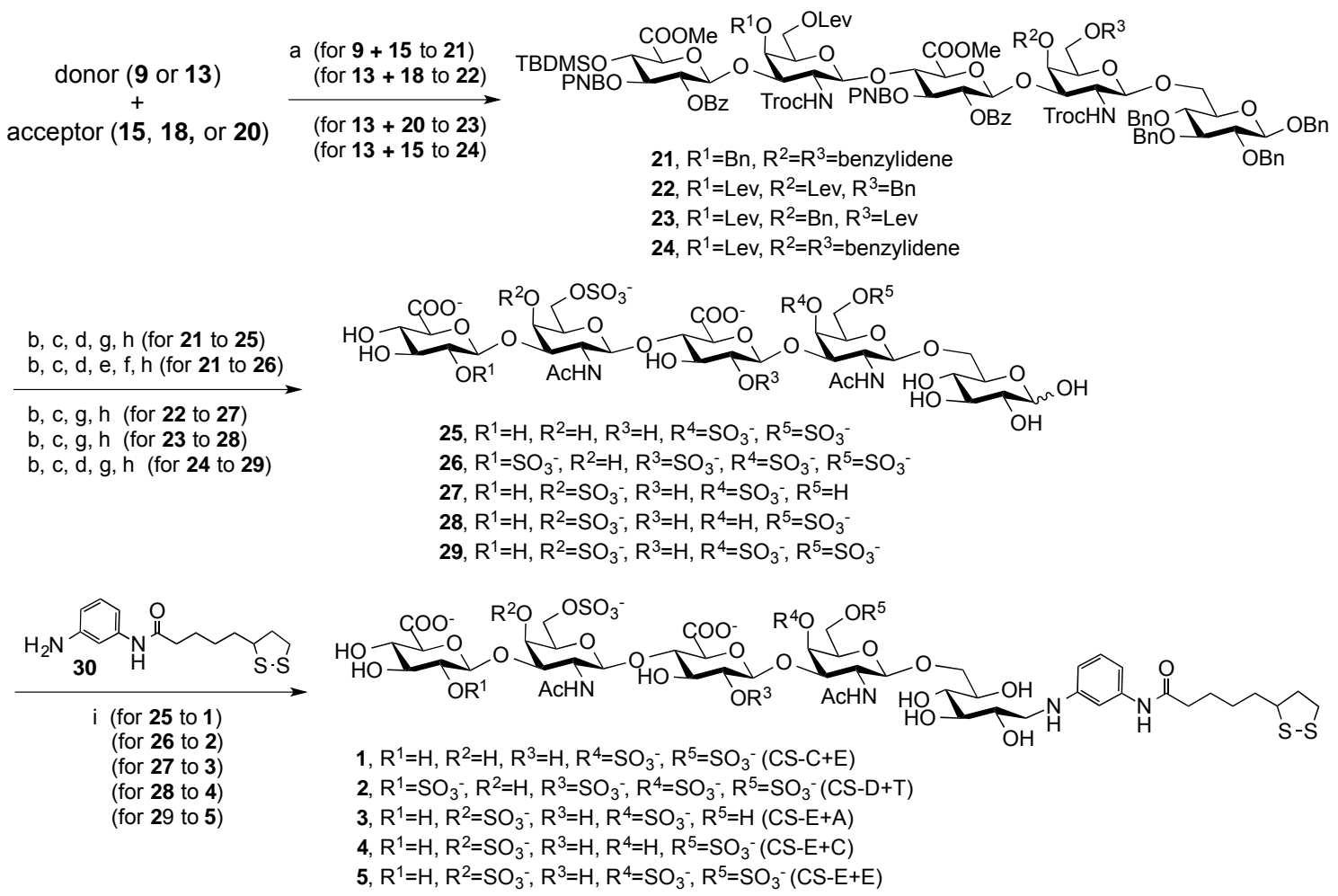

Scheme 3. (a) TMSOTf in $\mathrm{CH}_{2} \mathrm{Cl}_{2},-20{ }^{\circ} \mathrm{C}, 1$ h, $50 \%$ (2 steps) for $\mathbf{9}+\mathbf{1 5}$ to 21, $45 \%$ (2 steps) for $\mathbf{1 3}+\mathbf{1 8}$ to $\mathbf{2 2}$, $53 \%$ (2 steps) for $\mathbf{1 3}+\mathbf{2 0}$ to $\mathbf{2 3}, \mathbf{4 0 \%}$ (2 steps) for $\mathbf{1 3}+\mathbf{1 5}$ to $\mathbf{2 4}$; (b) $\mathrm{Zn}-\mathrm{Cu}$ couple in $\mathrm{AcOH}$, r.t., 1-3 h; $\mathrm{Ac}_{2} \mathrm{O}$ in Pyr., $0{ }^{\circ} \mathrm{C}, 2-4$ h, $47 \%$ (2 steps) for $\mathbf{2 1}$ to 25 and $\mathbf{2 6}$, $\mathrm{Zn}-\mathrm{Cu}$ couple in $\mathrm{AcOH} ; \mathrm{Ac}_{2} \mathrm{O}$ in Pyr., 36\% (2 steps) for $\mathbf{2 2}$ to 27, 38\% (2 steps) for $\mathbf{2 3}$ to $\mathbf{2 8}$, 31\% (2 steps) for 24 to 29; (c) hydrazine hydrate, AcOH in Pyr., r.t., 0.5-1 h. 99\% for 21 to 25 and 26, hydrazine hydrate, AcOH in Pyr., $63 \%$ for $\mathbf{2 2}$ to 27, $81 \%$ for $\mathbf{2 3}$ to $\mathbf{2 8}, \mathbf{8 5 \%}$ for $\mathbf{2 4}$ to 29; (d) TFA, $\mathrm{H}_{2} \mathrm{O}$ in $\mathrm{CH}_{2} \mathrm{Cl}_{2}, 0{ }^{\circ} \mathrm{C}, 2-4 \mathrm{~h}, 62 \%$ (recovery 30\%) for 21 to 25 and 26, $70 \%$ (recovery 25\%) for 24 to 29; (e) $\mathrm{NaOH}$ aq., $\mathrm{H}_{2} \mathrm{O}$ in THF, $0^{\circ} \mathrm{C}$, 4 h; $\mathrm{NaOH}$ aq. in $\mathrm{MeOH} / \mathrm{THF}$, $0{ }^{\circ} \mathrm{C}, 1 \mathrm{~d}, 92 \%$ (2 steps); (f) $\mathrm{SO}_{3} \bullet$ Pyr. in Pyr., $55^{\circ} \mathrm{C}, 10 \mathrm{~h}$; HF•pyridine in Pyr., $0{ }^{\circ} \mathrm{C}, 3-4 \mathrm{~d}$, 35\% (2 steps); (g) $\mathrm{SO}_{3} \bullet$ Pyr. in Pyr., 50-60 ${ }^{\circ} \mathrm{C}$, 4-16 h; HF•pyridine in Pyr., $0{ }^{\circ} \mathrm{C}, 3 \mathrm{~d}$; $\mathrm{NaOH}$ aq., $\mathrm{H}_{2} \mathrm{O}$ in THF, $0{ }^{\circ} \mathrm{C}, 2-4 \mathrm{~h}$; $\mathrm{NaOH}$ aq. in $\mathrm{MeOH} / \mathrm{THF}, 0^{\circ} \mathrm{C}, 1 \mathrm{~d}, 38 \%$ (4 steps) for 21 to 25, 35\% (4 steps) for 22 to 27, 41\% (4 steps) for $\mathbf{2 3}$ to $\mathbf{2 8}$, 36\% (4 steps) for $\mathbf{2 4}$ to $\mathbf{2 9}$; (h) Pd/C,

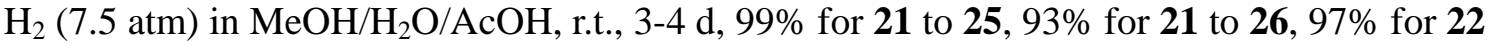


to 27, $\mathbf{8 6 \%}$ for $\mathbf{2 3}$ to $\mathbf{2 8}, \mathbf{9 7 \%}$ for $\mathbf{2 4}$ to $\mathbf{2 9}$; (i) $\mathbf{3 0}$ then $\mathrm{NaBH}_{3} \mathrm{CN}$ in $\mathrm{DMAc} / \mathrm{H}_{2} \mathrm{O} / \mathrm{AcOH}, 40{ }^{\circ} \mathrm{C}$, 3-4 d, 45\% (recovery: 50\%) for $\mathbf{2 5}$ to $\mathbf{1}, \mathbf{4 0 \%}$ (recovery: 52\%) for $\mathbf{2 6}$ to $\mathbf{2}$, 32\% (recovery: 65\%) for $\mathbf{2 7}$ to 3, 73\% (recovery: 20\%) for $\mathbf{2 8}$ to 4, 25\% (recovery: 60\%) for $\mathbf{2 9}$ to $\mathbf{5}$. 
Table 1. Kinetic parameters of the interaction between FGF-2 and chondroitin sulfate (CS) oligosaccharides.

\begin{tabular}{cccc}
\hline Unit structure & $k_{\mathrm{a}}\left(\mathrm{M}^{-1} \mathrm{~s}^{-1}\right)$ & $k_{\mathrm{d}}\left(\mathrm{s}^{-1}\right)$ & $K_{\mathrm{D}}(\mathrm{nM})$ \\
\hline CS-A & N.D.* & N.D.* & - \\
CS-C & $1.2 \times 10^{5}$ & $4.2 \times 10^{-3}$ & 36 \\
CS-D & $9.9 \times 10^{4}$ & $2.3 \times 10^{-3}$ & 23 \\
CS-E & $9.1 \times 10^{4}$ & $1.5 \times 10^{-3}$ & 16 \\
CS-T & $3.8 \times 10^{4}$ & $8.2 \times 10^{-4}$ & 21 \\
CS-C+D & N.D.* & N.D.* & - \\
CS-C+E & N.D.* & N.D.* & - \\
CS-D+D & $7.9 \times 10^{4}$ & $3.8 \times 10^{-3}$ & 48 \\
CS-D+T & $4.7 \times 10^{4}$ & $7.7 \times 10^{-4}$ & 16 \\
CS-E+A & $1.0 \times 10^{5}$ & $5.6 \times 10^{-4}$ & 5.6 \\
CS-E+C & $5.6 \times 10^{4}$ & $4.6 \times 10^{-4}$ & 8.2 \\
CS-E+D & $6.9 \times 10^{4}$ & $3.2 \times 10^{-4}$ & 4.7 \\
CS-E+E & $8.5 \times 10^{4}$ & $4.4 \times 10^{-4}$ & 5.2 \\
\hline
\end{tabular}

$*$ N.D., not determined due to weak interaction 


\section{Graphical Abstract}

Syntheses of chondroitin sulfate tetrasaccharide structures containing 4,6-disulfate patterns and analysis of their interaction with glycosaminoglycan-binding protein

Kento Miyachi, ${ }^{1}$ Masahiro Wakao, ${ }^{1 *}$ Yasuo Suda ${ }^{1,2 *}$

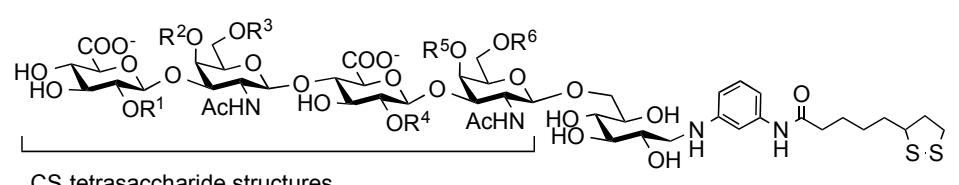

CS tetrasaccharide structures

CS-C+E (GlcA-GalNAc6S-GlcA-GalNAc4S6S)

CS-D+T (GlcA2S-GalNAc6S-GlcA2S-GalNAc4S6S)

CS-E+A (GlcA-GalNAc4S6S-GIcA-GalNAc4S)

CS-E+C (GlcA-GalNAc4S6S-GIcA-GalNAc6S)

CS-E+E (GIcA-GalNAc4S6S-GIcA-GalNAc4S6S) 